\title{
Some properties of Skorokhod metric on fuzzy sets
}

\author{
Huan Huang \\ Department of Mathematics, Jimei University, Xiamen 361021, China
}

\begin{abstract}
In this paper, we have our discussions on normal and upper semi-continuous fuzzy sets on metric spaces. The Skorokhod-type metric is stronger than the Skorokhod metric. It is found that the Skorokhod metric and the Skorokhodtype metric are equivalent on compact fuzzy sets. However, the Skorokhod metric and the Skorokhod-type metric need not be equivalent on $L_{p}$-integrable fuzzy sets. Based on this, we investigate relations between these two metrics and the $L_{p}$-type $d_{p}$ metric. It is found that the relations can be divided into three cases. On compact fuzzy sets, the Skorokhod metric is stronger than the $d_{p}$ metric. On $L_{p}$-integrable fuzzy sets, which take compact fuzzy sets as special cases, the Skorokhod metric is not necessarily stronger than the $d_{p}$ metric, but the Skorokhod-type metric is still stronger than the $d_{p}$ metric. On general fuzzy sets, even the Skorokhod-type metric is not necessarily stronger than the $d_{p}$ metric. We also show that the Skorokhod metric is stronger than the sendograph metric.
\end{abstract}

Keywords: Skorokhod metric; $L_{p}$ metric; Endograph metric; Sendograph metric; Hausdorff metric;

\section{Introduction}

Skorokhod metric on fuzzy sets has received deserving attentions. Joo and Kim [9] introduced the Skorokhod metric and the Skorokhod-type metric. They [9, 10] have proven that the Skorokhod metric and the Skorokhodtype metric are equivalent. Joo and Kim [10] have pointed out that the Skorokhod metric is stronger than the $d_{p}$ metric. Kim and Kim [11] have proven that the Skorokhod metric is stronger than the sendograph metric.

Email address: hhuangjy@126.com (Huan Huang) 
These results are obtained on the set of normal, upper semi-continuous and compact fuzzy sets on $\mathbb{R}^{m}$. Recently, Jardón, Sánchez and Sanchis [8] discussed the Skorokhod metric on compact fuzzy sets on metric space rather than just on $\mathbb{R}^{m}$. Here, a compact fuzzy set is a fuzzy set whose support set is compact, and a noncompact fuzzy set is a general fuzzy set whose support set may be compact or non-compact.

The $L_{p}$-type $d_{p}$ metric, endograph metric and sendograph metric are important and widely used metrics on fuzzy sets [1 6, 12 14]. Noncompact fuzzy sets, which take compact fuzzy sets as special cases, have received considerable attention from points of view of theory and practical applications $[3,6,12]$. The Euclidean space $\mathbb{R}^{m}$ is a kind of metric space. Of course, it is worthy to study fuzzy sets on general metric space.

So, it is important and natural to consider relation of the Skorokhod metric and the Skorokhod-type metric, and relation of these two metrics with the $d_{p}$ metric, the endograph metric and the sendograph metric, respectively, on noncompact fuzzy sets on metric space. In this paper, the discussions are carried out on normal and upper semi-continuous fuzzy sets on metric space. We assume that all the fuzzy sets mentioned in this paper are fuzzy sets of this type.

We confirm that the relations of the Skorokhod metric, the Skorokhodtype metric and the $d_{p}$ metric obtained in [9, 10] still hold on compact fuzzy sets.

However, we find that the relations of the Skorokhod metric, the Skorokhodtype metric and the $d_{p}$ metric on noncompact fuzzy sets are quite different from the case of compact fuzzy sets. This is the focus of our paper.

We show that the Skorokhod metric is stronger than the sendograph metric and the endograph metric.

The remainder of this paper is organized as follows. In Section 2, we recall some basic notions about fuzzy sets and various type of metrics on fuzzy sets. Then we introduce some subclasses of fuzzy sets and have some discussions related to metrics on fuzzy sets, which are useful in the sequel of this paper. In Section 3, we discuss the relation of the Skorokhod metric and the Skorokhodtype metric. In Sections 4, 5 and 6, we investigate relations between the two metrics, the Skorokhod metric and the Skorokhod-type metric, and the $d_{p}$ metric. The relation is divided into three cases. We mainly discuss one case in each section. In Section 7, we consider relation of the Skorokhod metric and the two metrics, the sendograph metric and the endograph metric. In Section 8, we give a simple example to answer some questions discussed 
recently. At last, we draw our conclusions in Section 9.

\section{Fuzzy sets and metrics on them}

In this section, we recall basic notions about fuzzy sets and metrics on fuzzy sets. Readers can refer to [1, 14] for more contents. We also introduce some subclasses of fuzzy sets and have some discussions related to metrics on fuzzy sets.

Let $(X, d)$ be a metric space and let $K(X)$ and $C(X)$ denote the set of all non-empty compact subsets of $X$ and the set of all non-empty closed subsets of $X$, respectively.

Metric $d_{a}$ is said to be stronger than metric $d_{b}$ on $X$, if the metric $d_{a}$ convergence can imply the metric $d_{b}$ convergence on $X$. Two metrics $d_{a}$ and $d_{b}$ are called to be equivalent on $X$ iff $d_{a}$ is stronger than $d_{b}$ on $X$ and $d_{b}$ is stronger than $d_{a}$ on $X$.

Let $F(X)$ denote the set of all fuzzy sets on $X$. A fuzzy set $u \in F(X)$ can be seen as a function $u: X \rightarrow[0,1]$. In this sense, a subset $S$ of $X$ can be seen as a fuzzy set

$$
\widehat{S}(x)= \begin{cases}1, & x \in S, \\ 0, & x \notin S .\end{cases}
$$

For $u \in F(X)$, let $[u]_{\alpha}$ denote the $\alpha$-cut of $u$, i.e.

$$
[u]_{\alpha}= \begin{cases}\{x \in X: u(x) \geq \alpha\}, & \alpha \in(0,1], \\ \operatorname{supp} u=\overline{\{u>0\}}, & \alpha=0 .\end{cases}
$$

For $u \in F(X)$, define

$$
\begin{gathered}
\text { end } u:=\{(x, t) \in X \times[0,1]: u(x) \geq t\}, \\
\text { send } u:=\{(x, t) \in X \times[0,1]: u(x) \geq t\} \cap\left([u]_{0} \times[0,1]\right) .
\end{gathered}
$$

end $u$ and send $u$ are called the endograph and the sendograph of $u$, respectively. The metric $\bar{d}$ on $X \times[0,1]$ is defined as

$$
\bar{d}((x, \alpha),(y, \beta))=d(x, y)+|\alpha-\beta| .
$$

Let $F_{U S C}^{1}(X)$ denote the set of all normal and upper semi-continuous fuzzy sets $u: X \rightarrow[0,1]$, i.e.,

$$
F_{U S C}^{1}(X):=\left\{u \in F(X):[u]_{\alpha} \in C(X) \text { for all } \alpha \in[0,1]\right\} .
$$


We use $H$ to denote the Hausdorff metric on $C(X)$ induced by $d$, i.e.,

$$
H(U, V)=\max \left\{H^{*}(U, V), H^{*}(V, U)\right\}
$$

for arbitrary $U, V \in C(X)$, where

$$
H^{*}(U, V)=\sup _{u \in U} d(u, V)=\sup _{u \in U} \inf _{v \in V} d(u, v) .
$$

If there is no confusion, we also use $H$ to denote the Hausdorff metric on $C(X \times[0,1])$ induced by $\bar{d}$.

The endograph metric $H_{\text {end }}$, the sendograph metric $H_{\text {send }}$, the supremum metric $d_{\infty}$, the Skorokhod metric $\rho_{0}$ and the Skorokhod-type metric $\rho_{1}$ can be defined on $F_{U S C}^{1}(X)$ as usual. The readers can see [1, 6, 8, 19, 12, 14, 15] for related contents.

For $u, v \in F_{U S C}^{1}(X)$,

$$
\begin{gathered}
H_{\text {end }}(u, v):=H(\text { end } u, \text { end } v), \\
H_{\text {send }}(u, v):=H(\operatorname{send} u, \operatorname{send} v), \\
d_{\infty}(u, v):=\sup \left\{H\left([u]_{\alpha},[v]_{\alpha}\right): \alpha \in[0,1]\right\},
\end{gathered}
$$

$\rho_{0}(u, v):=\inf \left\{\varepsilon:\right.$ there exists a $t$ in $T$ such that $D(t) \leq \varepsilon$ and $\left.d_{\infty}(u, t v) \leq \varepsilon\right\}$, $\rho_{1}(u, v):=\inf \left\{\varepsilon:\right.$ there exists a $t$ in $T$ such that $S(t) \leq \varepsilon$ and $\left.d_{\infty}(u, t v) \leq \varepsilon\right\}$

where $T$ is the class of strictly increasing, continuous mapping of $[0,1]$ onto $[0,1]$,

$$
\begin{gathered}
D(t):=\sup \{|t(\alpha)-\alpha|: \alpha \in[0,1]\}, \text { and } \\
S(t):=\sup \left\{\left|\ln \frac{t(\beta)-t(\alpha)}{\beta-\alpha}\right|: \alpha \neq \beta, \alpha, \beta \in[0,1]\right\}
\end{gathered}
$$

for each $t \in T$.

Remark 2.1. In [9], $\rho_{0}$ and $\rho_{1}$ are written as $d_{0}$ and $d_{1}$, respectively. It's also mentioned in [9] that $D(t)=D\left(t^{-1}\right)$ and $S(t)=S\left(t^{-1}\right)$ for all $t \in T$. In this paper, we call $\rho_{1}$ the "Skorokhod-type" metric.

Remark 2.2. It can be checked that for $u, u_{n}, n=1,2, \ldots$, in $F_{U S C}^{1}(X)$, $H_{\text {send }}\left(u_{n}, u\right) \rightarrow 0$ is equivalent to $H_{\text {end }}\left(u_{n}, u\right) \rightarrow 0$ and $H\left(\left[u_{n}\right]_{0},[u]_{0}\right) \rightarrow 0$. 
The $d_{p}$ metrics, $p \geq 1$, are widely used $L_{p}$-type metrics on fuzzy set spaces, which are very important from points of view of theory and applications.

The $d_{p}$ metric can be defined on $F_{U S C}^{1}(X)$ as usual, i.e.,

$$
d_{p}(u, v):=\left(\int_{0}^{1} H\left([u]_{\alpha},[v]_{\alpha}\right)^{p} d \alpha\right)^{1 / p}
$$

for $u, v \in F_{U S C}^{1}(X)$ when $H\left([u]_{\bullet},[v]_{\bullet}\right)$ is a measurable function on $[0,1]$.

Definition 2.3. We introduce the following subsets of $F_{U S C}^{1}(X)$, which will be useful in the sequel.

$F_{U S C B}^{1}(X):=\left\{u \in F_{U S C}^{1}(X):[u]_{0} \in K(X)\right\}$.

$F_{U S C G}^{1}(X):=\left\{u \in F_{U S C}^{1}(X):[u]_{\alpha} \in K(X)\right.$ when $\left.\alpha>0\right\}$.

$F_{U S C G}^{1}(X)^{p}:=\left\{u \in F_{U S C G}^{1}(X): d_{p}\left(u, \widehat{\left\{x_{0}\right\}}\right)=\left(\int_{0}^{1} H\left([u]_{\alpha},\left\{x_{0}\right\}\right)^{p} d \alpha\right)^{1 / p}<\right.$ $+\infty\}$, where $p \geq 1$ and $x_{0}$ is a point in $X$.

The definition of $F_{U S C G}^{1}(X)^{p}$ does not depend on the choice of $x_{0}$. Clearly,

$$
F_{U S C B}^{1}(X) \subset F_{U S C G}^{1}(X)^{p} \subset F_{U S C G}^{1}(X) \subset F_{U S C}^{1}(X) .
$$

Let $t \in T$. Then $t u \in F_{U S C B}^{1}(X)$ iff $u \in F_{U S C B}^{1}(X)$, and $u \in F_{U S C G}^{1}(X)$ iff $t u \in F_{U S C G}^{1}(X)$.

Let $\mathbb{R}^{m}, m \geq 1$, be the $m$-dimensional Euclidean space. Usually, we write $\mathbb{R}^{1}$ as $\mathbb{R}$ for simplicity. It can be checked that the following statements hold.

- For $u \in F_{U S C}^{1}(X)$ and $x_{0} \in X, H\left([u]_{\bullet},\left\{x_{0}\right\}\right)$ is a measurable function on $[0,1]$.

- For $u, v \in F_{U S C G}^{1}(X), H\left([u]_{\bullet},[v]_{\bullet}\right)$ is a measurable function on $[0,1]$.

- For $u, v \in F_{U S C}^{1}\left(\mathbb{R}^{m}\right), H\left([u]_{\bullet},[v]_{\bullet}\right)$ is a measurable function on $[0,1]$.

- There exists metric space $X$ and $u, v \in F_{U S C}^{1}(X)$ such that $H\left([u]_{\bullet},[v]_{\bullet}\right)$ is a non-measurable function on $[0,1]$.

Remark 2.4. In [8], the symbol $\mathcal{F}(X)$ is used to denote $F_{U S C B}^{1}(X)$.

The set of (compact) fuzzy numbers are denoted by $E^{m}$. It is defined as $E^{m}:=\left\{u \in F\left(\mathbb{R}^{m}\right):[u]_{\alpha}\right.$ is a nonempty compact convex subset of $\mathbb{R}^{m}$ for $\left.\alpha \in[0,1]\right\}$. 
Fuzzy numbers have attracted much attention from theoretical research and practical applications [1].

For $u, v \in F_{U S C}^{1}(X), H\left([u]_{\bullet},[v]_{\bullet}\right)$ could be a non-measurable function on $[0,1]$. So we introduce the following $d_{p}^{*}$ distance on $F_{U S C}^{1}(X)$.

The $d_{p}^{*}$ distance, $p \geq 1$, on $F_{U S C}^{1}(X)$, is defined as

$d_{p}^{*}(u, v):=\inf \left\{\left(\int_{0}^{1} f(\alpha)^{p} d \alpha\right)^{1 / p}: f\right.$ is a measurable function on $[0,1]$ with $\left.f(\cdot) \geq H\left([u]_{\bullet},[v]_{\bullet}\right)\right\}$

for $u, v \in F_{U S C}^{1}(X)$.

Theorem 2.5. $d_{p}^{*}$ is a metric on $F_{U S C}^{1}(X)$.

Proof. See Appendix.

Remark 2.6. Clearly, $d_{p}^{*}(u, v)=d_{p}(u, v)$ when $H\left([u]_{\bullet},[v]_{\bullet}\right)$ is a measurable function on $[0,1]$. So $d_{p}^{*}$ metric is an expansion of the $d_{p}$ metric on $F_{U S C}^{1}(X)$.

In the sequel, we don't distinguish between $d_{p}^{*}$ and $d_{p}$, both of which are written as $d_{p}$.

\section{Relation between $\rho_{0}$ and $\rho_{1}$ on $F_{U S C}^{1}(X)$}

Joo and Kim [9] have proven that the Skorokhod metric $\rho_{0}$ and the Skorokhod-type metric $\rho_{1}$ are equivalent on $E$. Further, they [10] pointed out that this relation still holds on $F_{U S C B}^{1}\left(\mathbb{R}^{m}\right)$.

In this section, we discuss the relation of the Skorokhod metric $\rho_{0}$ and the Skorokhod-type metric $\rho_{1}$ on $F_{U S C}^{1}(X)$. The $\rho_{1}$ convergence can still imply the $\rho_{0}$ convergence on $F_{U S C}^{1}(X)$. This statement can be deduced in the same way as the corresponding conclusion on $E$ in [9]. By establishing some lemmas, we point out that the method in [9] can also be used to prove the equivalence of $\rho_{0}$ and $\rho_{1}$ on $F_{U S C B}^{1}(X)$. However, we find that these two metrics are not necessarily equivalent on $F_{U S C G}^{1}(X)^{p}$, which is larger than $F_{U S C B}^{1}(X)$. A counterexample is given to show that the $\rho_{0}$ convergence need not imply the $\rho_{1}$ convergence on $F_{U S C G}^{1}(\mathbb{R})^{p}$.

Lemma 3.1. (i) $D(t) \rightarrow 0$ need not imply $S(t) \rightarrow 0$.

(ii) $S(t) \rightarrow 0$ implies that $D(t) \rightarrow 0$. 
Proof. (i) Example 3.2 provides a counterexample to show this statement.

(ii) This statement is from [9]. In fact, it can be deduced from the proof of Lemma 3.5 in [9].

Example 3.2. Consider $t_{a} \in T, a \in(0,1)$, defined as

$$
t_{a}(\xi)= \begin{cases}\sqrt{\xi}, & \xi \in[0, a], \\ \frac{1-\sqrt{a}}{1-a} \xi+\frac{\sqrt{a}-a}{1-a}, & \xi \in[a, 1] .\end{cases}
$$

It can be checked that $D\left(t_{a}\right)=|a-\sqrt{a}|$ for $a \leq \frac{1}{4}$ and $S\left(t_{a}\right) \equiv+\infty$ for each $a \in(0,1)$. Thus $D\left(t_{a}\right) \rightarrow 0$ as $a \rightarrow 0$. However $S\left(t_{a}\right) \not \rightarrow 0$ as $a \rightarrow 0$.

Proposition 3.3. $\rho_{1}$ is stronger than $\rho_{0}$ on $F_{U S C}^{1}(X)$.

Proof. The desired result follows immediately from Lemma 3.1 .

The following statement may be a known result. But we can not find the original literature which presents this fact.

Lemma 3.4. Let $\left\{u_{n}\right\} \subset K(X)$ satisfy $u_{1} \supseteq u_{2} \supseteq \ldots \supseteq u_{n} \supseteq \ldots$ Then $u=\bigcap_{n=1}^{+\infty} u_{n} \in K(X)$ and $H\left(u_{n}, u\right) \rightarrow 0$ as $n \rightarrow \infty$.

On the other hand, if $\left\{u_{n}\right\} \subset K(X), u_{1} \subseteq u_{2} \subseteq \ldots \subseteq u_{n} \subseteq \ldots$ and $u=\overline{\bigcup_{n=1}^{+\infty} u_{n}} \in K(X)$, then $H\left(u_{n}, u\right) \rightarrow 0$ as $n \rightarrow \infty$.

Proof. The desired result follows from the basic topology.

Suppose that $u \in F_{U S C G}^{1}(X), \alpha, \beta \in[0,1]$ and $\alpha<\beta$. The "variation" $w_{u}(\alpha, \beta)$ is defined as

$$
w_{u}(\alpha, \beta):=\sup \left\{H\left([u]_{\xi},[u]_{\eta}\right): \xi, \eta \in(\alpha, \beta]\right\} .
$$

The concept " $w_{u}(\alpha, \beta)$ " is from [9]. The following Lemma 3.5 is the version of fuzzy sets on metric space $(X, d)$ of Lemma 3.2 in [9].

Lemma 3.5. Suppose that $u \in F_{U S C B}^{1}(X)$. Given $\varepsilon>0$. Then there exist $\alpha_{1}, \alpha_{2}, \ldots, \alpha_{k}$ such that $0=\alpha_{1}<\alpha_{2}<\cdots<\alpha_{k}=1$ and

$$
w_{u}\left(\alpha_{i}, \alpha_{i+1}\right)<\varepsilon, i=1,2, \ldots, k-1 .
$$


Proof. Consider $[u](\cdot):[0,1] \rightarrow(K(X), H)$, which is the cut-function of $u$ and is defined by $[u](\alpha)=[u]_{\alpha}$.

From Lemma 3.4, $[u](\cdot)$ is left-continuous at $\alpha \in(0,1]$ and

$$
\lim _{\zeta \rightarrow h+} H\left([u]_{\zeta}, \overline{\bigcup\left\{[u]_{\alpha}: \alpha>h\right\}}\right)=0
$$

for $h \in[0,1)$.

The remainder proof can proceed similarly to the proof of Lemma 3.2 in [9].

Proposition 3.6. $\rho_{0}$ and $\rho_{1}$ are equivalent on $F_{U S C B}^{1}(X)$.

Proof. Using Lemma 3.5, the proof can proceed similarly to the proof of Lemma 3.7 and Theorem 3.8 in [9].

However, on $F_{U S C G}^{1}(X)^{p}$, which is larger than $F_{U S C B}^{1}(X), \rho_{0}$ and $\rho_{1}$ need not be equivalent. A counterexample is given in the following.

Example 3.7. Consider $u \in F_{U S C G}^{1}(\mathbb{R})$ defined as

$$
[u]_{\gamma}=\left[0, \gamma^{-0.4}\right]
$$

for all $\gamma>0$.

Clearly, $\rho_{0}\left(t_{a} u, u\right) \rightarrow 0$ as $a \rightarrow 0$, where $t_{a}$ is defined as in Example 3.2. However, it can be checked that $\rho_{1}\left(t_{a} u, u\right) \not \rightarrow 0$ as $a \rightarrow 0$. In fact, note that $S(t) \geq \ln \frac{\beta}{t^{-1} \beta}$ when $\beta \in(0,1]$, so for each $t \in T$ with $S(t)<+\infty$, there is a $K \geq 1$ such that $t^{-1}(\beta) \geq \beta / K$ for all $\beta \in[0,1]$. Thus for $a \in(0,1)$

$$
\begin{aligned}
& d_{\infty}\left(t u, t_{a} u\right) \\
& \quad \geq \sup _{\beta<a} H\left([t u]_{\sqrt{\beta}},\left[t_{a} u\right]_{\sqrt{\beta}}\right) \\
& \quad=\sup _{\beta<a} H\left([u]_{t^{-1} \sqrt{\beta}},[u]_{\beta}\right)=+\infty,
\end{aligned}
$$

and then $\rho_{1}\left(u, t_{a} u\right)=+\infty$.

Note that $u$ and $t_{a} u$ belong to $F_{U S C G}^{1}(\mathbb{R})^{1}$, so $\rho_{0}$ convergence need not imply $\rho_{1}$ convergence on $F_{U S C G}^{1}(\mathbb{R})^{1}$. A similar example can show that the $\rho_{0}$ convergence need not imply the $\rho_{1}$ convergence on $F_{U S C G}^{1}(\mathbb{R})^{p}$. 
Clearly, Example 3.7 indicates that $D(t) \rightarrow 0$ need not imply $S(t) \rightarrow 0$. The analysis in Example 3.7 also implies that $S\left(t_{a}\right)=+\infty$ for $a \in(0,1)$.

Wu, Zhang and Chen [15] proposed an example that a contraction whose Zadeh's extension is not a contraction under the Skorokhod metric and negatively answered the corresponding questions asked by Jardón, Sánchez and Sanchis [8]. In [7], we give a simple example to answer the questions.

\section{Relation between Skorokhod metric $\rho_{0}$ and $d_{p}$ metric on $F_{U S C B}^{1}(X)$}

In this section, it is shown that the Skorokhod metric $\rho_{0}$ is stronger than the $d_{p}$ metric on $F_{U S C B}^{1}(X)$. However, this is not the case with $F_{U S C G}^{1}(X)^{p}$, which is larger than $F_{U S C B}^{1}(X)$. A counterexample is given to show that the Skorokhod metric $\rho_{0}$ convergence need not imply the $d_{p}$ metric convergence on $F_{U S C G}^{1}(\mathbb{R})^{p}$.

Lemma 4.1. Let $u \in F_{U S C B}^{1}(X)$. Then $d_{p}(u, t u) \rightarrow 0$ as $D(t) \rightarrow 0$.

Proof. Given $\varepsilon>0$. From Lemma 3.5, there exist points $\alpha_{1}, \ldots, \alpha_{k}$ such that $0=\alpha_{1}<\alpha_{2}<\alpha_{k}=1$ and $w_{u}\left(\alpha_{l}, \alpha_{l+1}\right) \leq \varepsilon / 3$ for all $1 \leq l \leq k-1$.

Let

$$
M:=H\left([u]_{0},[u]_{1}\right)
$$

Then for each $t \in T$,

$$
\begin{aligned}
d_{p}(u, t u) & =\left(\int_{0}^{1} H\left([u]_{\alpha},[t u]_{\alpha}\right)^{p} d \alpha\right)^{1 / p} \\
& \leq \sum_{l=1}^{k-1}\left(\int_{\alpha_{l}}^{\alpha_{l+1}} H\left([u]_{\alpha},[t u]_{\alpha}\right)^{p} d \alpha\right)^{1 / p} \\
& \leq(k-1) M \cdot(2 D(t))^{1 / p}+\varepsilon / 3 .
\end{aligned}
$$

Thus, there is a $\zeta(\varepsilon)$ such that

$$
d_{p}(u, t u) \leq \varepsilon
$$

for all $t \in T$ with $D(t)<\zeta$.

Theorem 4.2. Suppose that $u \in F_{U S C B}^{1}(X), u_{n} \in F_{U S C}^{1}(X), n=1,2, \ldots$ If $\rho_{0}\left(u_{n}, u\right) \rightarrow 0$, then $d_{p}\left(u_{n}, u\right) \rightarrow 0$. 
Proof. Given $\varepsilon>0$. From Lemma 4.1 there is a $\zeta>0$ such that $d_{p}(u, t u)<$ $\varepsilon / 2$ for all $D(t)<\zeta$.

Since $\rho_{0}\left(u_{n}, u\right) \rightarrow 0$, then there exists an $N$ such that $\rho_{0}\left(u_{n}, u\right)<\eta=$ $\min \{\varepsilon / 2, \zeta\}$ for $n \geq N$. This means that, for each $n \geq N$, there is a $t_{n} \in T$ such that $D\left(t_{n}\right)<\eta$ and $d_{\infty}\left(u_{n}, t_{n} u\right)<\eta$.

So

$$
\begin{aligned}
d_{p}\left(u_{n}, u\right) & \leq d_{p}\left(u_{n}, t_{n} u\right)+d_{p}\left(t_{n} u, u\right) \\
& \leq \eta+\varepsilon / 2 \leq \varepsilon
\end{aligned}
$$

for all $n \geq N$.

The converse of the implication in Theorem 4.2 does not hold. $\left\{u_{n}\right\}$ and $u$ in Example 7.3 is a counterexample shows that the $d_{p}$ metric convergence need not imply the Skorokhod metric $\rho_{0}$ convergence on $F_{U S C B}^{1}(\mathbb{R})$.

Theorem 4.2 is not true if $F_{U S C B}^{1}(X)$ is replaced by $F_{U S C G}^{1}(X)^{p}$, which is larger than $F_{U S C B}^{1}(X)$. An example is given in the following to show that the Skorokhod metric $\rho_{0}$ convergence need not imply the $d_{p}$ metric convergence on $F_{U S C G}^{1}(\mathbb{R})^{p}$.

Example 4.3. Consider $t_{a, \theta},(a, \theta) \in(0,1) \times(0,1)$, defined as

$$
t_{a, \theta}(\xi)= \begin{cases}\xi^{\theta}, & \xi \in[0, a], \\ \frac{1-a^{\theta}}{1-a} \xi+\frac{a^{\theta}-a}{1-a}, & \xi \in[a, 1] .\end{cases}
$$

Then $t_{a, \theta} \in T$.

Consider $u \in F_{U S C G}^{1}(\mathbb{R})$ defined as

$$
[u]_{\gamma}=\left[0, \gamma^{-0.6}\right]
$$

for all $\gamma>0$. Then

$$
\begin{aligned}
d_{1}(u, \widehat{\{0\}}) & =\int_{0}^{1} H\left([u]_{\alpha},\{0\}\right) d \alpha \\
& =\int_{0}^{1} \alpha^{-0.6} d \alpha=2.5
\end{aligned}
$$

and therefore $u \in F_{U S C G}^{1}(\mathbb{R})^{1}$. 
Note that

$$
\begin{aligned}
d_{1}\left(\left(t_{a, \theta} u, \widehat{\{0\}}\right)\right. & =\int_{0}^{1} H\left(\left[t_{a, \theta} u\right]_{\gamma},\{0\}\right) d \gamma \\
& =\int_{0}^{a^{\theta}} \gamma^{-0.6 / \theta} d \gamma+\int_{a^{\theta}}^{1} H\left(\left[t_{a, \theta} u\right]_{\gamma},\{0\}\right) d \gamma
\end{aligned}
$$

and

$$
\begin{aligned}
d_{1}\left(t_{a, \theta} u, u\right) & =\int_{0}^{1} H\left(\left[t_{a, \theta} u\right]_{\gamma},[u]_{\gamma}\right) d \gamma \\
& \geq \int_{0}^{a^{\theta}} H\left(\left[t_{a, \theta} u\right]_{\gamma},[u]_{\gamma}\right) d \gamma \\
& =\int_{0}^{a^{\theta}}\left|\gamma^{-0.6 / \theta}-\gamma^{-0.6}\right| d \gamma
\end{aligned}
$$

thus there exist $a_{n} \rightarrow 0+$ and $\theta_{n} \rightarrow 0.6+$ such that

$$
\begin{gathered}
d_{1}\left(\left(t_{a_{n}, \theta_{n}} u, \widehat{\{0\}}\right)<+\infty\right. \\
d_{1}\left(t_{a_{n}, \theta_{n}} u, u\right)>0.5
\end{gathered}
$$

So $t_{a_{n}, \theta_{n}} u \in F_{U S C G}^{1}(\mathbb{R})^{1}, \rho_{0}\left(t_{a_{n}, \theta_{n}} u, u\right) \rightarrow 0$ and $d_{1}\left(t_{a_{n}, \theta_{n}} u, u\right) \nrightarrow \rightarrow 0$.

It can be shown by a similar example that the $\rho_{0}$ convergence need not imply the $d_{p}$ convergence on $F_{U S C G}^{1}(\mathbb{R})^{p}$.

\section{Relation between Skorokhod-type metric $\rho_{1}$ and $d_{p}$ metric on $F_{U S C G}^{1}(X)^{p}$}

In this section, we first discuss some basic properties of $u$ in $F_{U S C G}^{1}(X)^{p}$. Then we find a fact that for $u \in F_{U S C G}^{1}(X)^{p}, d_{p}(u, t u) \rightarrow 0$ as $S(t) \rightarrow 0$. Based on this, we show that the Skorokhod-type metric $\rho_{1}$ is stronger than the $d_{p}$ metric on $F_{U S C G}^{1}(X)^{p}$. A counterexample is given to show that the $d_{p}$ metric is not stronger than the Skorokhod-type metric $\rho_{1}$ on $F_{U S C G}^{1}(\mathbb{R})^{p} \backslash$ $F_{U S C B}^{1}(\mathbb{R})$.

Theorem 5.1. Suppose that $u \in F_{U S C G}^{1}(X)^{p}$ and $t \in T$. If $S(t)<+\infty$, then $t u \in F_{U S C G}^{1}(X)^{p}$. 
Proof. Suppose that $S(t)<+\infty$. Then there is a $K \geq 1$ such that $t^{-1}(\beta) \geq$ $\beta / K$ for all $\beta \in[0,1]$.

Thus

$$
\begin{aligned}
d_{p}\left(t u, \widehat{\left\{x_{0}\right\}}\right) & =\left(\int_{0}^{1} H\left([t u]_{\alpha},\left\{x_{0}\right\}\right)^{p} d \alpha\right)^{1 / p} \\
& \leq\left(\int_{0}^{1} H\left([u]_{\alpha / K},\left\{x_{0}\right\}\right)^{p} d \alpha\right)^{1 / p} \\
& =\left(\int_{0}^{1 / K} K H\left([u]_{\alpha},\left\{x_{0}\right\}\right)^{p} d \alpha\right)^{1 / p} \\
& \leq K^{1 / p}\left(\int_{0}^{1} H\left([u]_{\alpha},\left\{x_{0}\right\}\right)^{p} d \alpha\right)^{1 / p} \\
& =K^{1 / p} d_{p}\left(u, \widehat{\left\{x_{0}\right\}}\right) .
\end{aligned}
$$

So $t u \in F_{U S C G}^{1}(X)^{p}$.

Theorem 5.2. Suppose that $t \in T$ with $S(t)<+\infty$. Then $u \in F_{U S C G}^{1}(X)^{p}$ is equivalent to $t u \in F_{U S C G}^{1}(X)^{p}$.

Proof. Note that $u=t^{-1} t u$, thus the desired result follows from Theorem 5.1 .

If the condition $S(t)<+\infty$ is reduced to the condition $D(t)<+\infty$, then the conclusion in Theorem 5.1 does not hold. A counterexample is given in the following.

Example 5.3. Consider $t_{a} \in T, a \in(0,1)$, in Example 3.2 and $u$ given in Example 4.3. Then

$$
\begin{aligned}
d_{1}(u, \widehat{\{0\}}) & =\int_{0}^{1} H\left([u]_{\alpha},\{0\}\right) d \alpha \\
& =\int_{0}^{1} \alpha^{-0.6} d \alpha=2.5
\end{aligned}
$$

and therefore $u \in F_{U S C G}^{1}(\mathbb{R})^{1}$. 
Then

$$
\begin{aligned}
d_{1}\left(t_{0.3} u, \widehat{\{0\}}\right) & =\int_{0}^{1} H\left(\left[t_{0.3} u\right]_{\alpha},\{0\}\right) d \alpha \\
& \geq \int_{0}^{\sqrt{0.3}} H\left(\left[t_{0.3} u\right]_{\alpha},\{0\}\right) d \alpha \\
& =\int_{0}^{\sqrt{0.3}} \alpha^{-1.2} d \alpha=+\infty .
\end{aligned}
$$

So $t_{0.3} u \notin F_{U S C G}^{1}(\mathbb{R})^{1}$. In fact, it can be checked that $t_{a} u \notin F_{U S C G}^{1}(\mathbb{R})^{1}$ for $a \in(0,1)$.

Note that $D\left(t_{a}\right) \rightarrow 0$ as $a \rightarrow 0$. So even if $u \in F_{U S C G}^{1}(X)^{p}$ and $t \in T$ with $D(t)$ being less than any positive number required, $t u$ is still not necessarily in $F_{U S C G}^{1}(X)^{p}$.

Remark 5.4. Example 4.3 indicates that there exist $u \in F(\mathbb{R})$ and $t \in T$ such that $S(t)=+\infty$ and $u$ and $t u$ are both in $F_{U S C G}^{1}(\mathbb{R})^{p}$.

To show $\rho_{1}$ convergence can imply $d_{p}$ convergence on $F_{U S C G}^{1}(X)^{p}$, we need a fact that $d_{p}(u, t u) \rightarrow 0$ as $S(t) \rightarrow 0$ when $u \in F_{U S C G}^{1}(X)^{p}$. We begin with some lemmas.

Lemma 5.5. Suppose that $u \in F_{U S C G}^{1}(X)$. Given $h>0$ and $\varepsilon>0$. Then there exist $\alpha_{1}, \alpha_{2}, \ldots, \alpha_{k}$ and $\delta>0$ such that $h=\alpha_{1}<\alpha_{2}<\cdots<\alpha_{k}=1$,

$$
\begin{gathered}
w_{u}(h, h-\delta)<\varepsilon, \text { and } \\
w_{u}\left(\alpha_{i}, \alpha_{i+1}\right)<\varepsilon, i=1,2, \ldots, k-1 .
\end{gathered}
$$

Proof. Note that the cut-function $[u](\cdot):[0,1] \rightarrow(C(X), H)$ is left-continuous at $h$, the proof can proceed similarly to that of Lemma 3.5.

Lemma 5.6. Suppose that $u \in F_{U S C G}^{1}(X)^{p}$ and $h>0$. Then

$$
\left(\int_{h}^{1} H\left([u]_{\alpha},[t u]_{\alpha}\right)^{p} d \alpha\right)^{1 / p} \rightarrow 0
$$

as $D(t) \rightarrow 0$. 
Proof. By using Lemma 5.5, the proof can proceed similarly to the proof of Lemma 4.1.

The following important property of Lebesgue integral is useful in the proof of Theorem 5.7.

- Absolute continuity of Lebesgue integral. Suppose that $f$ is Lebesgue integrable on $E$, then for arbitrary $\varepsilon>0$, there is a $\delta>0$ such that $\int_{A} f d x<\varepsilon$ whenever $A \subseteq E$ and $m(A)<\delta$.

Theorem 5.7. Let $u \in F_{U S C G}^{1}(X)^{p}$. Then $d_{p}(u, t u) \rightarrow 0$ as $S(t) \rightarrow 0$.

Proof. Given $\varepsilon>0$. From the absolute continuity of Lebesgue integral, there is a $\theta>0$ such that for all $0 \leq h \leq \theta$

$$
\left(\int_{0}^{h} H\left([u]_{\alpha},\left\{x_{0}\right\}\right)^{p} d \alpha\right)^{1 / p} \leq \varepsilon / 3 .
$$

Choose $\xi>0$ satisfies that if $S(t)<\xi$ then $t^{-1}(\alpha)>\alpha / 1.1$ for all $\alpha \in[0,1]$. Thus, for all $0 \leq h \leq \theta$ and $t \in T$ with $S(t)<\xi$

$$
\begin{aligned}
& \left(\int_{0}^{h} H\left([t u]_{\alpha},\left\{x_{0}\right\}\right)^{p} d \alpha\right)^{1 / p} \\
& =\left(\int_{0}^{h} H\left([u]_{t^{-1}(\alpha)},\left\{x_{0}\right\}\right)^{p} d \alpha\right)^{1 / p} \\
& \leq\left(\int_{0}^{h} H\left([u]_{\alpha / 1.1},\left\{x_{0}\right\}\right)^{p} d \alpha\right)^{1 / p} \\
& =\left(\int_{0}^{h / 1.1} 1.1 H\left([u]_{\alpha},\left\{x_{0}\right\}\right)^{p} d \alpha\right)^{1 / p} \\
& \leq 1.1 \varepsilon / 3 .
\end{aligned}
$$

From Lemmas 3.1 and 5.6, there is a $\eta>0$ such that

$$
\left(\int_{\theta}^{1} H\left([u]_{\alpha},[t u]_{\alpha}\right)^{p} d \alpha\right)^{1 / p}<\varepsilon / 6
$$

when $S(t)<\eta$. 
So for $t \in T$ with $S(t)<\zeta=\min \{\xi, \eta\}$

$$
\begin{aligned}
& d_{p}(u, t u)=\left(\int_{0}^{1} H\left([u]_{\alpha},[t u]_{\alpha}\right)^{p} d \alpha\right)^{1 / p} \\
& \leq\left(\int_{0}^{\theta} H\left([u]_{\alpha},[t u]_{\alpha}\right)^{p} d \alpha\right)^{1 / p}+\left(\int_{\theta}^{1} H\left([u]_{\alpha},[t u]_{\alpha}\right)^{p} d \alpha\right)^{1 / p} \\
& \quad \leq\left(\int_{0}^{\theta} H\left([u]_{\alpha},\left\{x_{0}\right\}\right)^{p} d \alpha\right)^{1 / p}+\left(\int_{0}^{\theta} H\left([t u]_{\alpha},\left\{x_{0}\right\}\right)^{p} d \alpha\right)^{1 / p}+\left(\int_{\theta}^{1} H\left([u]_{\alpha},[t u]_{\alpha}\right)^{p} d \alpha\right)^{1 / p} \\
& \leq \varepsilon / 3+1.1 \varepsilon / 3+\varepsilon / 6<\varepsilon .
\end{aligned}
$$

Theorem 5.8. Suppose that $u \in F_{U S C G}^{1}(X)^{p}$ and $u_{n} \in F_{U S C}^{1}(X), n=$ $1,2, \ldots$ If $\rho_{1}\left(u_{n}, u\right) \rightarrow 0$, then $d_{p}\left(u_{n}, u\right) \rightarrow 0$.

Proof. The proof is similarly to that of Theorem 4.2 .

Given $\varepsilon>0$. From Theorem 5.7 there is a $\zeta>0$ such that $d_{p}(u, t u)<\varepsilon / 2$ for all $S(t)<\zeta$.

Since $\rho_{1}\left(u_{n}, u\right) \rightarrow 0$, then there exists an $N$ such that $\rho_{1}\left(u_{n}, u\right)<\nu=$ $\min \{\varepsilon / 2, \zeta\}$ for $n \geq N$. This means that, for each $n \geq N$, there is a $t_{n} \in T$ such that $S\left(t_{n}\right)<\nu$ and $d_{\infty}\left(u_{n}, t_{n} u\right)<\nu$.

So

$$
\begin{aligned}
d_{p}\left(u_{n}, u\right) & \leq d_{p}\left(u_{n}, t_{n} u\right)+d_{p}\left(t_{n} u, u\right) \\
& \leq \nu+\varepsilon / 2 \leq \varepsilon
\end{aligned}
$$

for all $n \geq N$.

Based on the results obtained in this paper, it can be seen that the conclusion in Theorems 5.7 and 5.8 can also be proved by using the the Lebesgue's Dominated Convergence Theorem.

In this section, we know that the Skorokhod-type metric $\rho_{1}$ is stronger than the $d_{p}$ metric on $F_{U S C G}^{1}(X)^{p}$. In Section 4 , we find that the Skorokhod metric $\rho_{0}$ is not necessarily stronger than the $d_{p}$ metric on $F_{U S C G}^{1}(X)^{p}$. These facts indicate that the Skorokhod metric $\rho_{0}$ is not necessarily equivalent to the Skorokhod-type metric $\rho_{1}$ on $F_{U S C G}^{1}(X)^{p}$. This is a conclusion in Section 3 .

The following example is given to show that the $d_{p}$ metric is not stronger than the Skorokhod-type metric $\rho_{1}$ on $F_{U S C G}^{1}(\mathbb{R})^{p} \backslash F_{U S C B}^{1}(\mathbb{R})$. 
Example 5.9. Consider $u \in F_{U S C G}^{1}(\mathbb{R})^{1} \backslash F_{U S C B}^{1}(\mathbb{R})$ defined as

$$
[u]_{\gamma}=\left[0, \gamma^{-0.6}\right]
$$

for all $\gamma>0$, and $t_{0.3, \theta}, \theta \in(0,1)$ defined in Example 4.3. Then $t_{0.3, \theta} u \in$ $F_{U S C G}^{1}(\mathbb{R})^{1} \backslash F_{U S C B}^{1}(\mathbb{R})$ for $\theta \in(0.6,1)$.

It can be checked that $\rho_{1}\left(u, t_{0.3, \theta_{n}} u\right) \not \rightarrow 0$ as $\theta_{n} \rightarrow 1-$.

On the other hand, it follows from the Lebesgue's Dominated Convergence Theorem that $d_{1}\left(u, t_{0.3, \theta_{n}} u\right) \rightarrow 0$ as $\theta_{n} \rightarrow 1-$.

So the $d_{1}$ metric is not stronger than the Skorokhod-type metric $\rho_{1}$ on $F_{U S C G}^{1}(\mathbb{R})^{1} \backslash F_{U S C B}^{1}(\mathbb{R})$. A similarly example can show that the $d_{p}$ metric is not stronger than the Skorokhod-type metric $\rho_{1}$ on $F_{U S C G}^{1}(\mathbb{R})^{p} \backslash F_{U S C B}^{1}(\mathbb{R})$.

Remark 5.10. From this example, we can see that even if a fuzzy set sequence is both $\rho_{0}$ convergence and $d_{p}$ convergence, it is not necessarily be $\rho_{1}$ convergence.

Let $v$ be defined as $[v]_{\gamma}=\left[0, \gamma^{-0.4 / p}\right], \gamma>0$, and let $t_{1 / n}$ be defined as in Example 3.2. Similarly, it can be checked that $\left\{t_{1 / n} v\right\}$ is a sequence in $F_{U S C G}^{1}(\mathbb{R})^{p} \backslash F_{U S C B}^{1}(\mathbb{R})$ which is $\rho_{0}$ convergence and $d_{p}$ convergence but is not $\rho_{1}$ convergence.

\section{Relation between Skorokhod-type metric $\rho_{1}$ and $d_{p}$ metric on $F_{U S C}^{1}(X)$}

In this section, we show that, unlike the case of $F_{U S C G}^{1}(X)^{p}$, the $\rho_{1}$ convergence is not necessarily the $d_{p}$ convergence on $F_{U S C}^{1}(X)$ by a counterexample.

The following example indicates that the $\rho_{1}$ convergence is not necessarily the $d_{p}$ convergence on $F_{U S C G}^{1}(X) \backslash F_{U S C G}^{1}(X)^{p}$.

Example 6.1. Consider $u \in F_{U S C G}^{1}(\mathbb{R}) \backslash F_{U S C G}^{1}(\mathbb{R})^{p}$ defined as

$$
[u]_{\gamma}=\left[0, \gamma^{-1.6}\right]
$$

for all $\gamma>0$.

Let $t^{a}, a \in(0,1)$, defined as

$$
t^{a}(\xi)= \begin{cases}(1+a) \xi, & \xi \in\left[0, \frac{1}{2}\right], \\ (1-a) \xi+a, & \xi \in\left[\frac{1}{2}, 1\right] .\end{cases}
$$

Then $t^{a} \in T, t^{a} u \in F_{U S C G}^{1}(\mathbb{R}) \backslash F_{U S C G}^{1}(\mathbb{R})^{p}$ for $a \in(0,1)$, and $\rho_{1}\left(u, t^{a} u\right) \rightarrow 0$ as $a \rightarrow 0$. 
On the other hand, for $a \in(0,1)$

$$
\begin{aligned}
d_{p}\left(u, t^{a} u\right) & =\left(\int_{0}^{1} H\left([u]_{\gamma},\left[t^{a} u\right]_{\gamma}\right)^{p} d \gamma\right)^{1 / p} \\
& \geq\left(\int_{0}^{1 / 2} H\left(\left[0, \gamma^{-1.6}\right], \quad\left[0,\left((1+a)^{-1} \gamma\right)^{-1.6}\right]\right)^{p} d \gamma\right)^{1 / p} \\
& =+\infty
\end{aligned}
$$

So the $\rho_{1}$ convergence need not imply the $d_{p}$ convergence on $F_{U S C G}^{1}(\mathbb{R}) \backslash F_{U S C G}^{1}(\mathbb{R})^{p}$.

It can also be checked that the $\rho_{1}$ convergence is not necessarily the $d_{p}$ convergence on $F_{U S C}^{1}(X) \backslash F_{U S C G}^{1}(X)$.

From the results in Sections 4, 5 and 6, the following statements are true for $u, u_{n}$ in $F_{U S C}^{1}(X), n=1,2, \ldots$.

(i) If $u \in F_{U S C B}^{1}(X)$, then $\rho_{0}\left(u_{n}, u\right) \rightarrow 0$ can imply $d_{p}\left(u_{n}, u\right) \rightarrow 0$.

(ii) If $u \in F_{U S C G}^{1}(X)^{p}$, then $\rho_{1}\left(u_{n}, u\right) \rightarrow 0$ can imply $d_{p}\left(u_{n}, u\right) \rightarrow 0$. However $\rho_{0}\left(u_{n}, u\right) \rightarrow 0$ need not imply $d_{p}\left(u_{n}, u\right) \rightarrow 0$.

(iii) If $u \in F_{U S C}^{1}(X)$, then $\rho_{1}\left(u_{n}, u\right) \rightarrow 0$ need not imply $d_{p}\left(u_{n}, u\right) \rightarrow 0$.

\section{Relation between Skorokhod metric and sendograph metric on $F_{U S C}^{1}(X)$}

In this section, it is found that the Skorokhod metric is stronger than the sendograph metric and the endograph metric on $F_{U S C}^{1}(X)$. The sendograph metric is stronger than the endograph metric (see Section 21). A counterexample is given to show that the sendograph metric convergence need not imply the Skorokhod metric convergence on $F_{U S C B}^{1}(\mathbb{R})$.

For $u \in F_{U S C}^{1}(X)$, the symbol $P_{0}(u)$ is used to denote the set $\{\alpha \in(0,1)$ : $\left.\lim _{\beta \rightarrow \alpha} H\left([u]_{\beta},[u]_{\alpha}\right) \neq 0\right\}$.

Theorem 7.1. Let $u_{n}, u, n=1,2, \ldots$, be fuzzy sets in $F_{U S C}^{1}(X)$. If $\rho_{0}\left(u_{n}, u\right) \rightarrow$ 0 , then

(i) $H\left(\left[u_{n}\right]_{0},[u]_{0}\right) \rightarrow 0$,

(ii) $H\left(\left[u_{n}\right]_{1},[u]_{1}\right) \rightarrow 0$,

(iii) $H_{\text {end }}\left(u_{n}, u\right) \rightarrow 0$,

(iv) $H_{\text {send }}\left(u_{n}, u\right) \rightarrow 0$, and

(v) $H\left(\left[u_{n}\right]_{\alpha},[u]_{\alpha}\right) \rightarrow 0$ for all $\alpha \in(0,1) \backslash P_{0}(u)$. 
Proof. Note that $t(0)=0$ and $t(1)=1$ for each $t \in T$. So

$$
\begin{aligned}
\rho_{0}(u, v) & \geq H\left([u]_{0},[v]_{0}\right), \\
\rho_{0}(u, v) & \geq H\left([u]_{1},[v]_{1}\right)
\end{aligned}
$$

for all $u, v \in F_{U S C}^{1}(X)$ and therefore (i) and (ii) are true.

To prove (iii). Given $\varepsilon>0$. Since $\rho_{0}\left(u_{n}, u\right) \rightarrow 0$, then there exists $N$, for each $n \geq N$, there is a $t_{n} \in T$ such that $d_{\infty}\left(t_{n} u_{n}, u\right)<\varepsilon / 2$ and $D\left(t_{n}\right)<\varepsilon / 2$. Thus

$$
\begin{aligned}
& H^{*}\left(\text { end } u, \text { end } u_{n}\right) \\
& \quad=\sup \left\{d\left((x, \alpha), \text { end } u_{n}\right):(x, \alpha) \in \text { end } u\right\} \\
& \quad \leq \sup \left\{H\left([u]_{\alpha},\left[u_{n}\right]_{t_{n}^{-1}(\alpha)}\right)+\varepsilon / 2:(x, \alpha) \in \text { end } u\right\} \\
& \quad \leq d_{\infty}\left(t_{n} u_{n}, u\right)+\varepsilon / 2 \\
& \quad \leq \varepsilon
\end{aligned}
$$

and

$$
\begin{aligned}
& H^{*}\left(\text { end } u_{n}, \text { end } u\right) \\
& \quad=\sup \left\{d((x, \alpha), \text { end } u):(x, \alpha) \in \text { end } u_{n}\right\} \\
& \quad \leq \sup \left\{H\left(\left[u_{n}\right]_{\alpha},[u]_{t_{n}(\alpha)}\right)+\varepsilon / 2:(x, \alpha) \in \text { end } u_{n}\right\} \\
& \quad \leq d_{\infty}\left(t_{n} u_{n}, u\right)+\varepsilon / 2 \\
& \quad \leq \varepsilon
\end{aligned}
$$

From the arbitrariness of $\varepsilon>0$,

$$
H_{\text {end }}\left(u_{n}, u\right)=\max \left\{H^{*}\left(\text { end } u \text {, end } u_{n}\right), H^{*}\left(\text { end } u_{n} \text {, end } u\right)\right\} \rightarrow 0 .
$$

So (iii) is true.

The proof of (iv) is very similar to that of (iii).

To prove (v), suppose that $\alpha \in(0,1) \backslash P_{0}(u)$. Given $\varepsilon>0$. There exists a $\delta>0$ such that

$$
H\left([u]_{\beta},[u]_{\alpha}\right)<\varepsilon / 2
$$

for all $\beta \in(\alpha-\delta, \alpha+\delta)$.

From $\rho_{0}\left(u_{n}, u\right) \rightarrow 0$, we know that there is an $N$ such that $\rho_{0}\left(u_{n}, u\right)<$ $\zeta=\min \{\delta, \varepsilon / 2\}$ for all $n \geq N$. This means that for each $n \geq N$, there is a $t_{n}$ such that

$$
d_{\infty}\left(u_{n}, t_{n} u\right)<\zeta \text { and } D\left(t_{n}\right)<\zeta
$$


By (11) and (2), for all $n \geq N$,

$$
\begin{aligned}
H & \left(\left[u_{n}\right]_{\alpha},[u]_{\alpha}\right) \\
& \leq H\left(\left[u_{n}\right]_{\alpha},[u]_{t_{n}^{-1}(\alpha)}\right)+H\left([u]_{t_{n}^{-1}(\alpha)},[u]_{\alpha}\right) \\
& \leq d_{\infty}\left(u_{n}, t_{n} u\right)+H\left([u]_{t_{n}^{-1}(\alpha)},[u]_{\alpha}\right) \\
& <\zeta+\varepsilon / 2 \leq \varepsilon .
\end{aligned}
$$

From the arbitrariness of $\left.\varepsilon>0, H\left[u_{n}\right]_{\alpha},[u]_{\alpha}\right) \rightarrow 0$. So $(\mathrm{v})$ is true.

Remark 7.2. The (iv) in Theorem 7.1 can also be deduced from the relation of sendograph metric and endograph metric (see Section 21) and the (i) and (iii) in Theorem 7.1 .

Theorem 7.1 indicates that the Skorokhod metric convergence can imply the sendograph metric convergence on $F_{U S C}^{1}(X)$. However, the converse implication does not hold. The following is an example of a sequence in $F_{U S C B}^{1}(\mathbb{R})$ which is sendograph metric convergence but is not Skorokhod metric convergence.

Example 7.3. Consider

$$
u(x)= \begin{cases}1, & x=0 \\ \frac{1}{2}, & x \in(0,2] \\ 0, & x \notin[0,2]\end{cases}
$$

and

$$
u_{n}(x)= \begin{cases}1-\frac{1}{2} x^{1 / n}, & x \in[0,1], \\ \frac{1}{2}\left(1-(x-1)^{n}\right), & x \in[1,2], \quad n=1,2, \ldots \\ 0, & x \notin[0,2],\end{cases}
$$

So $u$ and $u_{n}, n=1,2, \ldots$ are in $F_{U S C B}^{1}(\mathbb{R})$, and

$$
[u]_{\alpha}= \begin{cases}\{0\}, & \alpha \in(1 / 2,1] \\ {[0,2],} & \alpha \in[0,1 / 2]\end{cases}
$$

and

$$
\left[u_{n}\right]_{\alpha}= \begin{cases}{\left[0,(2-2 \alpha)^{n}\right],} & \alpha \in[1 / 2,1], \\ {\left[0,1+(1-2 \alpha)^{1 / n}\right],} & \alpha \in[0,1 / 2] .\end{cases}
$$

Note that $\left[u_{n}\right]_{1 / 2} \equiv[0,1]$, so for all $n=1,2, \ldots$,

$$
\rho_{0}\left(u_{n}, u\right) \geq 1
$$


In fact it can be checked that $\rho_{0}\left(u_{n}, u\right) \equiv 1$.

On the other hand, since

$$
H\left(\left[u_{n}\right]_{\alpha},[u]_{\alpha}\right) \rightarrow 0 \text { for all } \alpha \in[0,1] \backslash\left\{\frac{1}{2}\right\} .
$$

Thus by Theorem 6.4 in [6], $H_{\text {end }}\left(u_{n}, u\right) \rightarrow 0$, and then $H_{\text {send }}\left(u_{n}, u\right) \rightarrow 0$. So $\left\{u_{n}\right\}$ and $u$ satisfy statements (i)-(v) in Theorem 7.1. But $\rho_{0}\left(u_{n}, u\right) \not \rightarrow 0$.

In addition, we can see that $d_{p}\left(u_{n}, u\right) \rightarrow 0$. So this example also indicates that the $d_{p}$ metric convergence need not imply the Skorokhod metric $\rho_{0}$ convergence on $F_{U S C B}^{1}(\mathbb{R})$. This fact can also be derived from the conclusions in [6], see Section 8 .

\section{Conclusion}

In this paper, we first discuss the relation between the Skorokhod metric $\rho_{0}$ and the Skorokhod-type metric $\rho_{1} . \rho_{1}$ is stronger than $\rho_{0}$ on $F_{U S C}^{1}(X)$. It is found that $\rho_{1}$ is equivalent to $\rho_{0}$ on $F_{U S C B}^{1}(X)$, and that $\rho_{1}$ is not necessarily equivalent to $\rho_{0}$ on $F_{U S C G}^{1}(X)^{p}$.

Then we investigate relation between these two metrics and $d_{p}$ metric. It is found that the compactness of $\alpha$-cuts and the integrability of fuzzy sets play important roles. On $F_{U S C B}^{1}(X)$, the Skorokhod metric $\rho_{0}$ is stronger than the $d_{p}$ metric. On $F_{U S C G}^{1}(X)^{p}$, the Skorokhod-type metric $\rho_{1}$ is still stronger than the $d_{p}$ metric, however the Skorokhod metric $\rho_{0}$ is not necessarily stronger than the $d_{p}$ metric. On $F_{U S C}^{1}(X)$, even the Skorokhod-type metric $\rho_{1}$ is not necessarily stronger than the $d_{p}$ metric. We also show that the Skorokhod metric $\rho_{0}$ is stronger than the sendograh metric on $F_{U S C}^{1}(X)$.

Our recent results on level decomposition properties of the endograph metric can immediately imply that $H_{\text {send }}\left(u_{n}, u\right) \rightarrow 0$ is equivalent to $d_{p}\left(u_{n}, u\right) \rightarrow$ 0 and $H\left(\left[u_{n}\right]_{0},[u]_{0}\right) \rightarrow 0$ on $F_{U S C B}^{1}\left(\mathbb{R}^{m}\right)$ (see the end of Section 6 or Theorem 6.4 in [6]). So the statement in [11] that the Skorokhod metric $\rho_{0}$ is stronger than the sendograph metric on $F_{U S C B}^{1}\left(\mathbb{R}^{m}\right)$ can be derived from the statement in [10] that the Skorokhod metric $\rho_{0}$ is stronger than the $d_{p}$ metric on $F_{U S C B}^{1}\left(\mathbb{R}^{m}\right)$. We find some interesting relations among the metrics on fuzzy sets which will be presented in the future work.

\section{Appendix A. The proof of Theorem 2.5}

Proof. To prove that $d_{p}^{*}$ is a metric, we need to show that, for all $u, v, w$ in $F_{U S C}^{1}(X)$, 
(i) $d_{p}^{*}(u, v) \geq 0$ and $d_{p}^{*}(u, v)=0$ is equivalent to $u=v$,

(ii) $d_{p}^{*}(u, v)=d_{p}^{*}(v, u)$, and

(iii) $d_{p}^{*}(u, v) \leq d_{p}^{*}(u, w)+d_{p}^{*}(v, w)$.

(i) Obviously $d_{p}^{*}(u, v) \geq 0$. Now we show that $d_{p}^{*}(u, v)=0$ is equivalent to $u=v$.

If $u=v$, then $d_{p}^{*}(u, v)=d_{p}(u, v)=0$.

If $u \neq v$, then there is an $\alpha>0$ such that $[u]_{\alpha} \neq[v]_{\alpha}$. We claim that outer measure $m^{*}$ of the set $S:=\left\{\beta \in[0, \alpha]:[u]_{\beta} \neq[v]_{\beta}\right\}$ is greater than 0 . We proceed by contradiction. Suppose $m^{*}(S)=0$. Then $[\alpha-\varepsilon, \alpha) \nsubseteq S$ for each $\varepsilon>0$, and therefore there is a sequence $\left\{\alpha_{n}, n=1,2, \ldots\right\}$ with $\alpha_{n} \in\left[\alpha-\frac{1}{n}, \alpha\right)$ and $[u]_{\alpha_{n}}=[v]_{\alpha_{n}}$. This contradicts with $[u]_{\alpha} \neq[v]_{\alpha}$.

Since $m^{*}(S)>0$, then there exists $k>0$ and $\varepsilon_{0}>0$ such that $m^{*}\left(\left\{H\left([u]_{\bullet},[v]_{\bullet}\right)>\right.\right.$ $1 / k\})>\varepsilon_{0}$. So if $f$ is a measurable function on $[0,1]$ with $f(\cdot) \geq H\left([u]_{\bullet},[v]_{\bullet}\right)$, then $m(f>1 / k)>\varepsilon_{0}$. This implies that $d_{p}^{*}(u, v)>0$.

(ii) holds obviously.

(iii) If $f$ is a measurable function on $[0,1]$ with $f(\cdot) \geq H\left([u]_{\bullet},[w]_{\bullet}\right)$ and $g$ is a measurable function on $[0,1]$ with $g(\cdot) \geq H\left([v]_{\bullet},[w]_{\bullet}\right)$, then $f+g$ is a measurable function on $[0,1]$ with $(f+g)(\cdot) \geq H\left([u]_{\bullet},[v]_{\bullet}\right)$. So

$$
\begin{aligned}
d_{p}^{*}(u, v) & \leq\left(\int_{0}^{1}(f(\alpha)+g(\alpha))^{p} d \alpha\right)^{1 / p} \\
& \leq\left(\int_{0}^{1} f(\alpha)^{p} d \alpha\right)^{1 / p}+\left(\int_{0}^{1} g(\alpha)^{p} d \alpha\right)^{1 / p} .
\end{aligned}
$$

From the arbitrariness of $f$ and $g$,

$$
d_{p}^{*}(u, v) \leq d_{p}^{*}(u, w)+d_{p}^{*}(v, w)
$$

[1] P. Diamond, P. Kloeden, Metric Spaces of Fuzzy Sets, World Scientific, Singapore, 1994

[2] D. Dubois, H. Prade (Eds.), Fundamentals of Fuzzy Sets, vol 1 of the Handbooks of Fuzzy Sets, Kluwer, Boston, Mass, 2000

[3] P. Grzegorzewski, Metrics and orders in space of fuzzy numbers, Fuzzy Sets Syst. 97 (1998) 83-94 
[4] P. Grzegorzewski, Nearest interval approximation of a fuzzy number, Fuzzy Sets Syst. 130 (2002) 321-330.

[5] H. Huang, C. Wu, Characterizations of compact sets in fuzzy set spaces with $L_{p}$ metric, Fuzzy Sets Syst. 330 (2018) 16-40

[6] H. Huang, Characterizations of endograph metric and $\Gamma$-convergence on fuzzy sets, Fuzzy Sets Syst. 350 (2018) 55-84

[7] H. Huang, Some notes on convergence structures on fuzzy sets, 1669 in Mathematics in National Science and Technology Library, https://www.nstl.gov.cn/index.html, 2019-04-23

[8] D. Jardón, I. Sánchez, M. Sanchis, Some questions about Zadeh's extension on metric spaces, Fuzzy Sets Syst. (2019), https://doi.org/10.1016/j.fss.2018.10.019

[9] S.Y. Joo, Y.K. Kim, The Skorokhod topology on space of fuzzy numbers, Fuzzy Sets Syst. 111 (2000) 497-501.

[10] S.Y. Joo, Y.K. Kim, Topological properties on the space of fuzzy sets, J. Math. Anal. Appl. 246 (2000) 576-590

[11] D. S. Kim, Y. K. Kim, Some properties of a new metric on the space of fuzzy numbers, Fuzzy Sets Syst. 145 (2004) 395-410

[12] P.E. Kloeden, T. Lorenz, A Peano theorem for fuzzy differential equations with evolving membership grade, Fuzzy Sets Syst. 280 (2015) $1-26$

[13] J. Kupka, On approximations of Zadeh's extension principle, Fuzzy Sets Syst. 283 (2016) 26-39

[14] C. Wu, M. Ma, The Basic of Fuzzy Analysis (in Chinese), National Defence Industry press, Beijing, 1991

[15] X. Wu, X. Zhang, G. Chen, Answers to some questions about Zadeh's extension principle on metric spaces, Fuzzy Sets Syst. (2019) https://doi.org/10.1016/j.fss.2019.03.011 